\title{
Micro-stability and transport modelling of internal transport barriers on JET
}

\author{
X. Garbet ${ }^{1}$, Y. Baranov ${ }^{2}$, G. Bateman ${ }^{3}$, S. Benkadda ${ }^{4}$, P. Beyer ${ }^{4}$, \\ R. Budny ${ }^{5}$, F. Crisanti ${ }^{6}$, B. Esposito ${ }^{6}$, C. Figarella $^{4}$, C. Fourment ${ }^{1}$, \\ P. Ghendrih ${ }^{1}$, F. Imbeaux ${ }^{1}$, E. Joffrin ${ }^{1}$, J. Kinsey ${ }^{3}$, A. Kritz ${ }^{3}$, \\ X. Litaudon ${ }^{1}$, P. Maget $^{1}$, P. Mantica ${ }^{7}$, D. Moreau ${ }^{1}$, Y. Sarazin ${ }^{1}$,

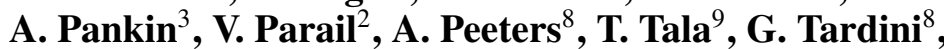 \\ A. Thyagaraja ${ }^{2}$, I. Voitsekhovitch ${ }^{4}$, J. Weiland ${ }^{10}, \mathbf{R}$. Wolf ${ }^{11}$ and \\ JET EFDA contributors ${ }^{\mathrm{a}}$ \\ ${ }^{1}$ Association EURATOM-CEA, CEA Cadarache, 13108 St Paul-Lez-Durance, France \\ ${ }^{2}$ EURATOM/UKAEA, Culham Science Centre, Abingdon OX14 3DB, UK \\ ${ }^{3}$ Lehigh University Physics Department, 16 Memorial Drive East, Bethlehem, PA 18015 , \\ USA \\ ${ }^{4}$ LPIIM, Centre Universitaire de Saint-Jerôme, 13397 Marseille Cedex 20, France \\ ${ }^{5}$ PPPL, Princeton University, PO Box 451, Princeton, NJ 08543, USA \\ ${ }^{6}$ Associazione EURATOM-ENEA sulla Fusione, Via Enrico Fermi 27, 00044 Frascati, Italy \\ ${ }^{7}$ Istituto di Fisica del Plasma CNR-EURATOM, via Cozzi 53, 20125 Milano, Italy \\ ${ }^{8}$ MPI für Plasmaphysik, EURATOM-Assoziation, D-8046 Garching bei München, Germany \\ ${ }^{9}$ Association EURATOM-TEKES, VTT CTIP, FIN-02044 VTT, Finland \\ ${ }^{10}$ Chalmers University of Technology and EURATOM-VR Assocation, S-41296 Göteborg, \\ Sweden \\ ${ }^{11}$ Institut für Plasmaphysik, Association EURATOM/FZJ, D-52425 Jülich \\ E-mail: garbet@cea.fr
}

Received 15 November 2002, accepted for publication 9 July 2003

Published 28 August 2003

Online at stacks.iop.org/NF/43/975

\begin{abstract}
The physics of internal transport barrier (ITB) formation in JET has been investigated using micro-stability analysis, profile modelling and turbulence simulations. The calculation of linear growth rates shows that magnetic shear plays a crucial role in the formation of the ITB. Shafranov shift, ratio of the ion to electron temperature, and impurity content further improve the stability. This picture is consistent with profile modelling and global fluid simulations of electrostatic drift waves. Turbulence simulations also show that rational $q$ values may play a special role in triggering an ITB. The same physics also explains how double internal barriers can be formed.
\end{abstract}

PACS numbers: 52.65.Tt, 52.55.fa, 52.35.Ra

\section{Introduction}

Internal transport barriers (ITBs) in tokamak plasmas are considered as a promising way to achieve steady-state plasmas with good confinement properties in a fusion reactor. A crucial question is whether it will be possible to produce an ITB in a next-step device with a reasonable amount of power. Once a barrier is triggered, a self-amplifying process takes place, where increasing gradients produce $E \times B$ velocity shear and Shafranov shift large enough to further decrease the turbulent transport. This paper is, however, focused on the

a See annex of Pamela J. et al 2002 Proc.19th on Fusion energy IAEA (Lyon, 2002) (Vienna: IAEA). question of barrier initiation. Many experimental results in JET point towards the safety factor profile as a key ingredient. In particular, the power threshold is clearly lower when the magnetic shear is reversed. However, other mechanisms like Shafranov shift stabilization, impurity content or density peaking may play a role. One aim of this paper is to apply and compare various models and techniques on a common set of JET plasmas. Micro-stability analysis, profile modelling and turbulence simulations are used for this purpose. This paper also tackles a challenging class of transport barriers that are sensitive to low order rational surfaces. Their role has been recently confirmed in JET reversed shear plasmas, thanks to the observation of Alfvèn cascades in coincidence with barrier formation. In particular, strong barriers are often 
triggered when $q_{\min }$ crosses 2 or 3 . Surprisingly, when $q_{\min }$ further decreases with time and falls below $q=2$, the barrier sometimes splits. Two internal barriers then coexist and are tied to the $q=2$ magnetic surfaces. Existing models are confronted with this puzzling behaviour.

\section{Brief description of JET ITBs}

The physics that is usually invoked for explaining the triggering and self-sustainment of an ITB is a mixture of turbulence suppression via $E \times B$ velocity shear and linear stabilization of drift waves. The magnetic shear is often considered as the main reason for improved stability. Two mechanisms have been identified: a decrease of the interchange drive [1], which is more prominent at negative shear, and a rarefaction of resonant surfaces that occurs at zero shear [2]. The reduction in turbulent transport comes from a decrease of the drive and/or smaller correlation lengths. Other parameters are also likely play some role, for example, the Shafranov shift (also called $\alpha$ effect, $\alpha=-q^{2} R \mathrm{~d} \beta / \mathrm{d} r$ ), density gradient, impurity content and ratio of the ion to electron temperature. A common way to assess this stabilization is to compare the $E \times B$ shear rate $\gamma_{E}$ to a maximum linear growth rate $\gamma_{\text {lin }}$ [3-6].

Two operational criteria can be built on the basis of this simple rule. We consider here ion temperature gradient (ITG) modes and collisionless TEM modes (the collision frequency is well below the curvature drift frequency in JET ITBs). A set of fluid-like equations describing these modes is given in the appendix. They are similar to those used in the Weiland model [7]. The instability is of the interchange type (see equation (A.6)), i.e. assuming $T_{\mathrm{e}} \approx T_{\mathrm{i}}$, the linear growth rate $\gamma_{\text {lin }}$ is of the form $c_{\mathrm{s}} /\left(R L_{T_{\mathrm{e}}}\right)^{1 / 2}$ up to a function of plasma parameters $\left(c_{\mathrm{s}}\right.$ is the sound speed and $L_{T_{\mathrm{e}}}$ the electron temperature gradient length). The diamagnetic part of the rotational shear rate reads $\gamma_{\mathrm{E}} \approx \rho_{\mathrm{S}} c_{\mathrm{S}} / L_{T_{\mathrm{e}}}^{2}$. The condition $\gamma_{\mathrm{E}}>\gamma_{\text {lin }}$ then transforms into $\left(R / L_{T_{\mathrm{e}}}\right)^{1 / 2} \rho_{\mathrm{s}} / L_{T_{\mathrm{e}}}>$ cte. For simplicity, this criterion for a transition is transformed into $\rho_{\mathrm{T}}^{*}=\rho_{\mathrm{s}} / L_{T_{\mathrm{e}}}>\rho_{T_{\text {crit }}}^{*}$. In principle, $\rho_{T_{\text {crit }}}^{*}$ depends on the magnetic shear, $\alpha$, and the Mach number (which comes into play via the contribution of the toroidal velocity to the shear flow), and possibly the density gradient and impurity content. In practice, an analysis of the JET database shows that this criterion works well when choosing a constant value $\rho_{T_{\text {crit }}}^{*}=0.014$ [8]. Another criterion corresponds to a 'loss of stiffness'. Stiffness here means that the temperature gradient length (for ions or electrons) is close to a threshold value $R / L_{\mathrm{T}}=R / L_{T_{\text {crit }}}$. This hypothesis is still under investigation at JET. Ion cyclotron modulation experiments with mode conversion in L mode show the existence of a threshold for electrons [9]. For ions evidence has been obtained from steadystate profiles in $\mathrm{L}$ and $\mathrm{H}$ modes [10]. A natural definition of an ITB then corresponds to a region were the threshold is well above the $\mathrm{L}$ mode value. This leads to a criterion of the form $R / L_{\mathrm{T}}>R / L_{T_{\text {crit }}}$ for the formation of the ITB. This rule can be written as a condition on the ratio of core to edge temperature. A large class of ion ITBs was found to satisfy this criterion using a critical value $R / L_{T_{\text {crit }}} \approx 6[10]$.

\section{Micro-stability analysis of JET plasmas with an ITB}

Several fluid and kinetic stability codes have been used to calculate the growth rates of ITG modes and trapped electron modes (TEM) [7, 11-13]. The various techniques used to calculate the linear growth rates and $E \times B$ velocity shear are summarized in table 1. Details can be found in references [14-21]. These models have been compared on the same JET pulse \#51976.

This pulse is a transient ITB with high performance that was analysed in detail by Challis et al [22]. The $q$ profile is reversed early in the discharge using a current pre-forming phase with lower hybrid current drive (LHCD). An electron barrier appears early in the plasma at $t \approx 1.5 \mathrm{~s}$, after LHCD is applied. An ion barrier develops at $t=4 \mathrm{~s}$ after neutral beam injection (NBI) is switched on. Both electron and ion barriers are strongly amplified at $t \approx 6 \mathrm{~s}$ (see figure 1 ). The $q$ profile shown in figure 2 is from a TRANSP run [12]. All groups have used the Hahm-Burrell definition [5] of the $E \times B$ shear rate. However, the calculation procedures were different (see table 1), thus leading to substantial differences (figure 2). Part of this discrepancy comes from the different ways of fitting the data. Also, the Hahm-Burrell expression may be calculated locally (the major radius being the radial coordinate), or by using flux coordinates. Thus, the mapping of experimental data on the equilibrium is a source of uncertainty. The result of the stability analysis at $t=6 \mathrm{~s}$, before the barrier strengthens, is shown in figure 2 . Note that a barrier already exists at that

Table 1. List of models used to analyse JET transport barriers

\begin{tabular}{|c|c|c|}
\hline Name & Growth rate & $E_{\mathrm{r}}$ calculation \\
\hline Weiland [7] & $\begin{array}{l}\text { Fluid ITG } \\
\text { (Weiland [7]) }\end{array}$ & NCLASS [19] \\
\hline GS2 [12] & $\begin{array}{l}\text { Gyrokinetic flux tube } \\
\text { ITG/TEM (GS2) [16] }\end{array}$ & NCLASS [19] \\
\hline Rogister [13] & Rogister model [15] & Kim model [20] \\
\hline Kine0 [14] & $\begin{array}{l}\text { Variational gyrokinetic } \\
\text { ITG/TEM } \\
\text { (KINEZERO) [18] }\end{array}$ & Kim model [20] \\
\hline
\end{tabular}

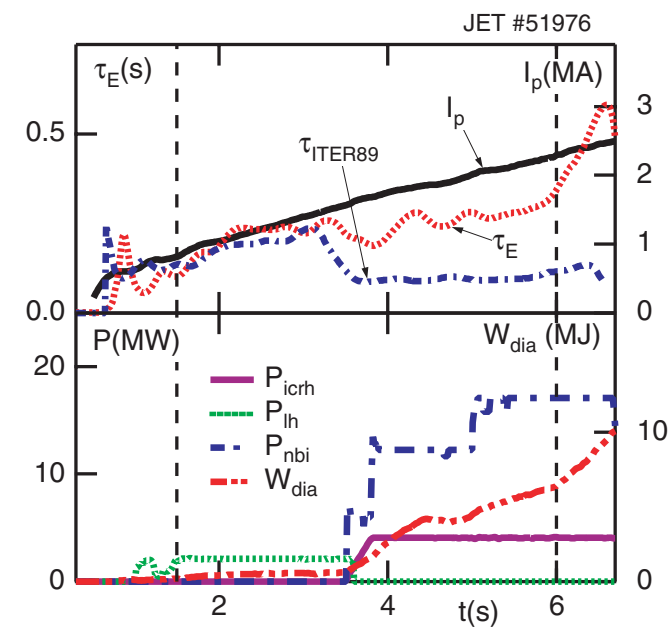

Figure 1. Time history of the JET pulse \#51976. Top panel: plasma current $I_{\mathrm{p}}$, energy confinement time $\tau_{\mathrm{E}}$ and ITER89 scaling law. Lower panel: additional power and energy content. 

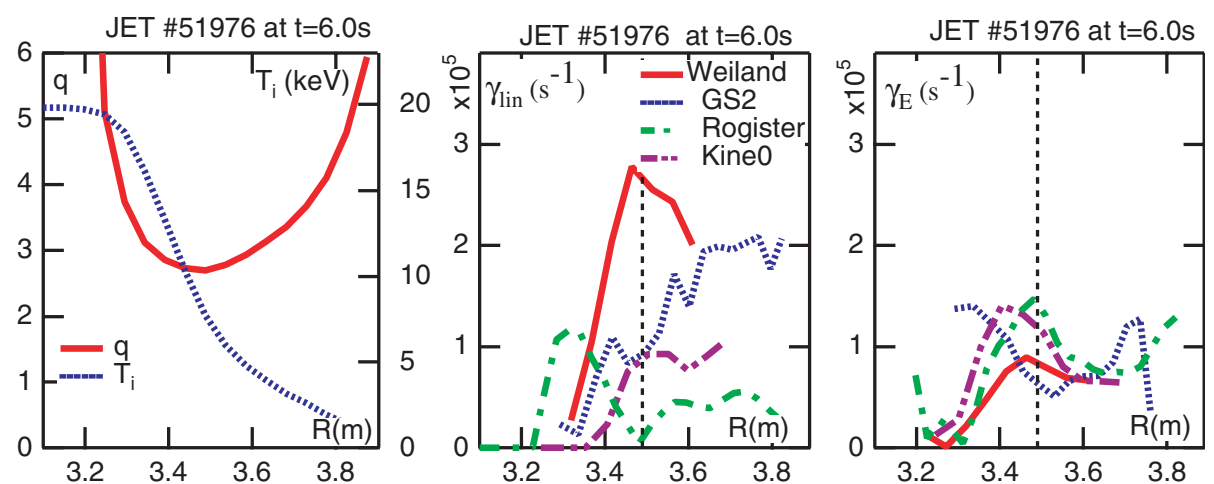

Figure 2. Profiles of safety factor and ion temperature, linear growth rates and velocity shear rate of JET pulse \#51976 at $t=6 \mathrm{~s}$.

time, so that the velocity shear rate is already large. Three models do predict stabilization, whereas the Weiland model predicts growth rates that are too large to be overcome by the velocity shear rate. However, this model uses a ballooning representation that is not valid in the vicinity of $q=q_{\min }$. Using the Rogister model [15] instead gives a better agreement.

Explaining the electron barrier onset at $t=1.5 \mathrm{~s}$ is more difficult, since no measurement of ion temperature and safety factor is available at the beginning of the pulse. A transition due to the $E \times B$ velocity shear or $\alpha$ stabilization alone seems difficult to justify. Before the transition, $\alpha$ is of the order of 0.1 . The estimated value of the velocity shear rate $\gamma_{\mathrm{E}}$ is low $\left(\approx 10^{4} \mathrm{~s}^{-1}\right)$ as compared to a typical value of $\gamma_{\text {lin }}$, unless a burst of localized rotational shear occurs (this possibility is analysed in section 6). This finding is in line with previous studies where a blip of NBI was done in the preheat phase, allowing a better calculation of the velocity profile $[23,24]$. Thus, a decrease of the growth rate has to be invoked to explain this transition. In practice, most models rely essentially on the magnetic shear to trigger the barrier. This effect is less marked when using the GS2 code, which predicts a transition at $t \approx 5 \mathrm{~s}$ [12]. In the latter case, the stabilization is due to the combined contributions of the negative magnetic shear, Shafranov shift and impurity content. No obvious difference is seen between negative and zero magnetic shear. The Rogister model favours low magnetic shear, confirmed by a recent analysis of the JET database [25], whereas the GS2 (flux tube) code predicts that negative shear is more favourable.

\section{Profile modelling of JET ITBs}

JET ITB plasmas have been modelled using several available transport models: Mixed Bohm-gyroBohm (B/gB) [26, 27], multi-mode (MMM) [28], and Weiland [7, 29] models. B/gB models have been implemented in the JETTO and CRONOS codes. The main differences between the two codes come from the LHCD modules (FRTC in JETTO and Delphine in CRONOS). Moreover, the stabilization by magnetic shear and $E \times B$ velocity is implemented in different ways. Namely, the JETTO code enforces a global decrease of the diffusivity in the region where $\gamma_{\mathrm{E}}>0.68 \gamma_{\mathrm{ITG}}(s-0.14)$ [26], where $\gamma_{\mathrm{ITG}}$ is approximated by $v_{T_{\mathrm{i}}} / R\left(v_{T_{\mathrm{i}}}\right.$ is the ion thermal velocity). The CRONOS local uses a smoother and local reduction of the diffusivity $1 /\left[1+\exp \left(20\left(0.05+\gamma_{\mathrm{E}} / \gamma_{\mathrm{ITG}}-s\right)\right)\right]$, with a growth rate $\gamma_{\text {ITG }}$ given by Newman et al [17]. This exercise

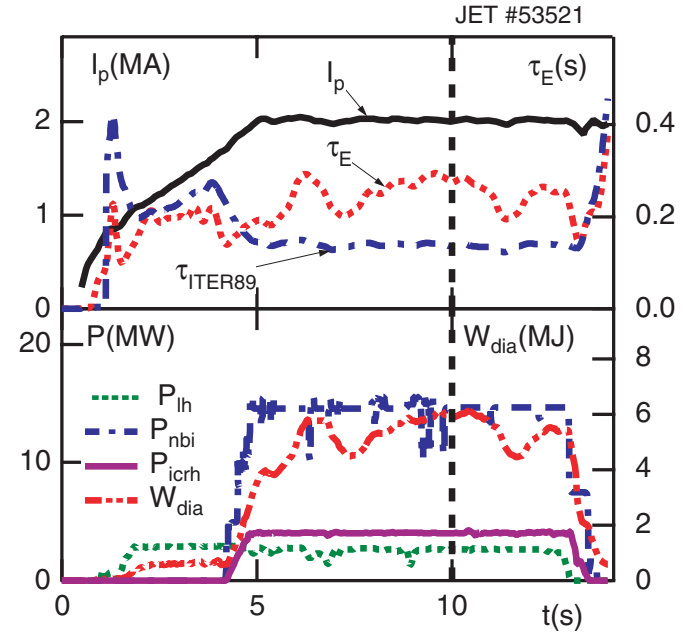

Figure 3. Time history of pulse \#53521. Top panel: plasma current $I_{\mathrm{p}}$, energy confinement time $\tau_{\mathrm{E}}$ and ITER89 scaling law. Lower panel: additional power and energy content.

was carried out for pulse \#51976 (see section 2) and the quasisteady-state ITB \#53521 [30] with similar results.

The time history of pulse \#53521 is shown in figure 3. An important difference with \#51976 is that the LHCD is present throughout the plasma duration. The whole pulse was simulated. A comparison is shown in figure 4 in the quasisteady-state phase at $t=10 \mathrm{~s}$. This is a good test since the final state depends sensitively on the time history. The transport models that predict a strong decrease of the diffusivities for negative or zero magnetic shear reach the best agreement. Interestingly, the two simulations using the mixed $\mathrm{B} / \mathrm{gB}$ model show some differences. This is due to the different current drive modules and the differences in the nature of the transition (local or global). This sensitivity of the current profile is not surprising since the onset of the barrier is mainly due to the magnetic shear, whereas the velocity shear rate is small at the transition. This result is in line with the findings of the stability analysis (section 3). Later on in the pulse, the velocity shear rate becomes increasingly important for maintaining the barrier and moving its location outward. Another interesting feature is that the MMM is in better agreement with the data than the Weiland model. This was unexpected since the Weiland model is part of the MMM. The reason is that the HamaguchiHorton criterion [6] $\gamma_{\mathrm{E}} / s>\gamma_{\text {lin }}$ ( $s$ is the magnetic shear) was 

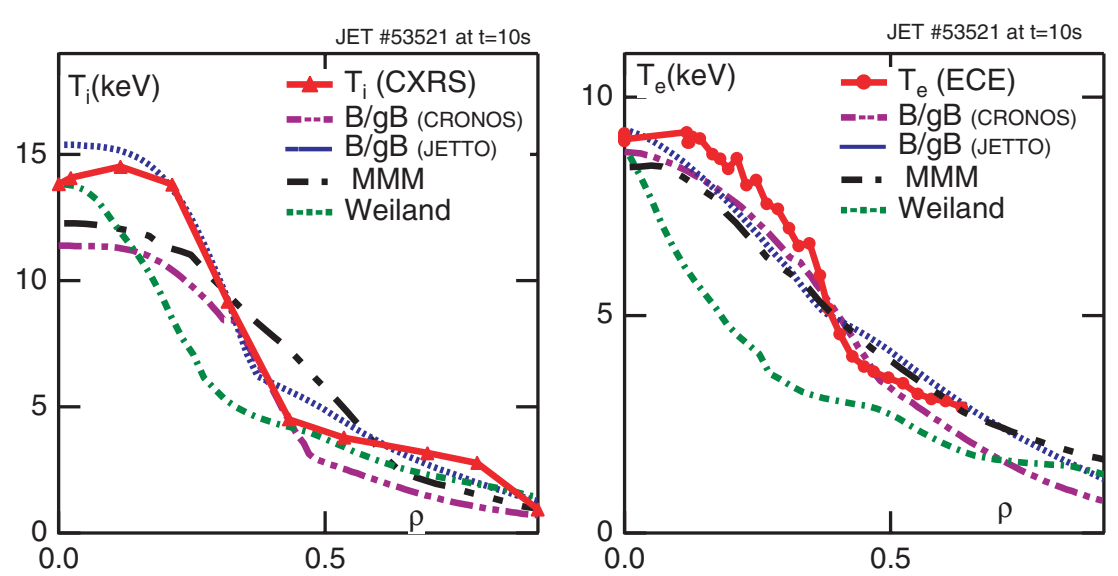

Figure 4. Profile modelling comparison for pulse \#53521 at $t=10 \mathrm{~s}$.
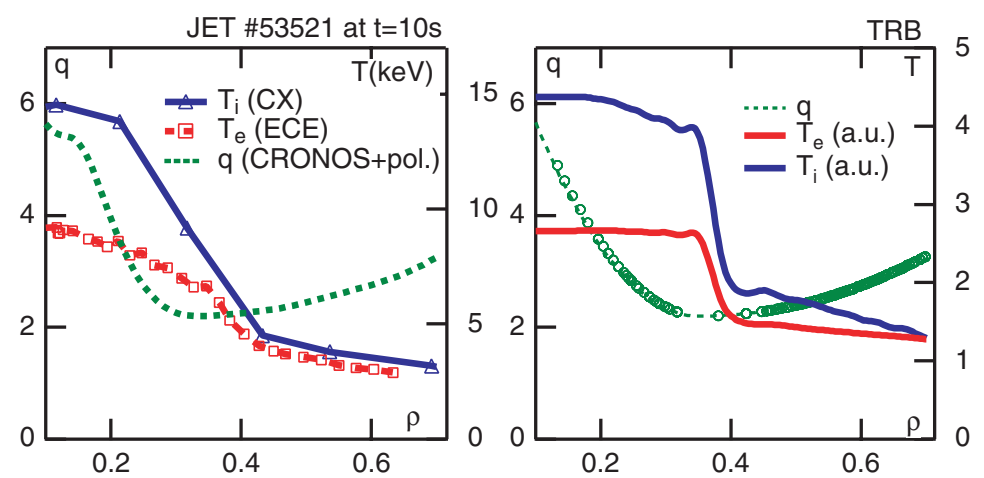

Figure 5. Left panel: experimental profiles of safety factor, electron and ion temperature of the JET pulse \#53521 at $t=10 \mathrm{~s}(q$ profile from [30]). Right panel: turbulence simulation of a barrier with the same $q$ profile. Circles are the positions of $k_{\theta} \rho_{\mathrm{s} 0}<1$ resonant surfaces on the $q$ profile.

used in the MMM analysis whereas the Hahm-Burell criterion $\gamma_{\mathrm{E}}>\gamma_{\text {lin }}$ was used for the simulation using the Weiland model. Using the Hamaguchi-Horton criterion introduces a strong dependence on the magnetic shear in the modelling, thus palliating the weak dependence of the linear growth rate on the magnetic shear in the Weiland model. This result is also in line with the linear stability analysis. The Weiland model predicts an ITB formation but the density gradient seems to be the key ingredient in this case [29]. Thus, although many results point in the direction of the magnetic shear as being mainly responsible for the transition to an ITB, other mechanisms cannot be excluded.

\section{Turbulence simulations of JET ITBs}

Global fluid simulations of electrostatic ITG/TEM modes (TRB code $[31,32]$ ) have been run for several JET plasmas. The equations that have been solved are described in the appendix. The model covers collisionless TEM modes and slab and toroidal ITG modes. All simulations show the importance of the magnetic shear for the onset of the barrier. However, different mechanisms are involved for ions and electrons. Although high wave number ITG modes are stabilized by negative shear, the main reason for the onset of an ion barrier is the formation of a gap in the density of rational surfaces at low wave numbers close to the minimum of safety factor $q_{\min }$ (see figure 5). Close to a low order rational number, e.g. $q_{\min }=2$, there exists indeed a substantial layer without any resonant surface such that $k_{\theta} \rho_{\mathrm{s}}<1$. The width of this layer can actually be estimated analytically. Assuming $q_{\min }=m_{0} / n_{0}$ and choosing $q$ as a radial coordinate, the distance between the resonant surface associated to an $(m, n)$ mode and the surface $q=q_{\min }$ is $\Delta q=\left|m n_{0}-n m_{0}\right| / n_{0} n$. For a given toroidal wave number $n$, the minimum value of $\Delta q$ over all values of $m$ is $1 / n_{0} n$. Harmonics of $\left(m_{0}, n_{0}\right)$ are excluded since these modes resonate at $q=q_{\text {min }}$. The ratio $1 / n_{0} n$ can be made as low as needed by increasing the toroidal wave number. However, restricting the toroidal wave number to the domain of unstable ITG/TEM modes $n q \rho_{\mathrm{s}} / r<1$, it is found that this minimum distance remains finite, $\Delta q_{\text {gap }} \approx q_{\min } \rho_{\mathrm{s}} /\left(r_{q_{\min }} n_{0}\right)$. If $q_{\min }=m_{0} / n_{0}$ is chosen very close, but slightly above a simple rational number, i.e. $q_{\min }=m_{0} / n_{0}+\varepsilon$, with $\varepsilon \ll 1$, this distance has to be multiplied by 2 since each side of $q=q_{\text {min }}$ has to be accounted for. This width can be recast into a distance in radial coordinate by assuming a parabolic profile around $q_{\min }$, i.e. $q=q_{\min }+q_{\min }^{\prime \prime}\left(r-r_{q_{\min }}\right)^{2} / 2$. One finds

$$
d_{\text {gap }}=2\left[\frac{q_{\min } \rho_{\mathrm{s}}}{q_{\min }^{\prime \prime} r_{q_{\min }} n_{0}}\right]^{1 / 2}
$$

Taking as an example $r_{q_{\min }} q_{\min }^{\prime \prime} / q_{\min }=0.5$ and an ion Larmor radius of $10^{-3} \mathrm{~m}$ leads to a gap of the order of $0.1 \mathrm{~m}$ for $q_{\min }=2\left(m_{0}=2, n_{0}=1\right)$. A barrier appears when this 
gap is larger than a turbulence correlation length. We note, however, that this argument holds if resonant modes only play a dominant role in turbulence. The possible onset of nonresonant modes is, therefore, a central issue. Once an ion barrier is produced, its position and width are controlled by rotational shear. Electrons are sensitive to both negative and zero magnetic shear. Obviously TEMs are also affected by a gap in the resonant surfaces, even if they do not need an overlap with adjacent resonant surfaces to be unstable. TEMs are also affected by a negative magnetic shear because of the reversal of the trapped electron curvature drift that decreases the instability drive. Full stabilization of TEM modes is expected for $s<-\frac{3}{8}$ in this model.

Simulations of an actual ITB in JET indicate that all mechanisms are involved, depending on the $q$ profile. An example for pulse \#53521 is shown in figure 5. Regarding the turbulence characteristics, these simulations agree with those previously carried on for resistive ballooning mode turbulence [33]. In the latter case, transport barriers were produced with an externally imposed velocity shear. In particular, a strong decrease of electric potential fluctuations is always observed, whereas the decrease of density or pressure fluctuation amplitude is small in weak barriers. Thus, the level

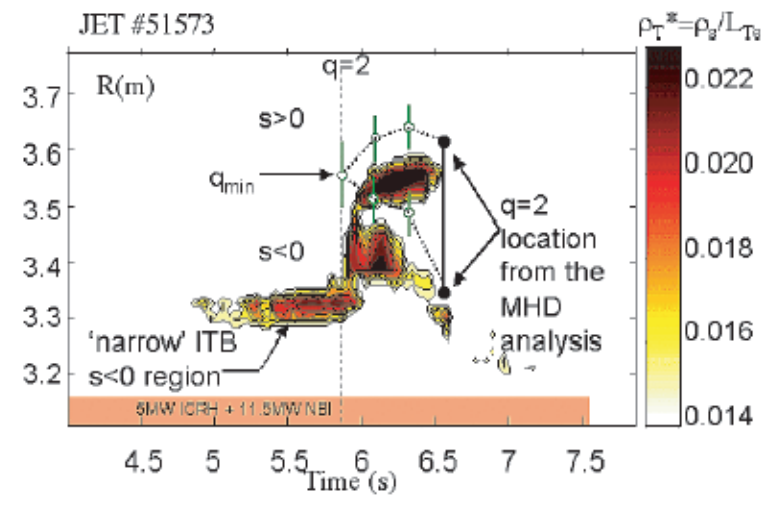

Figure 6. Contours of $\rho_{\mathrm{T}}^{*}$ of pulse 51573 (from [34]).

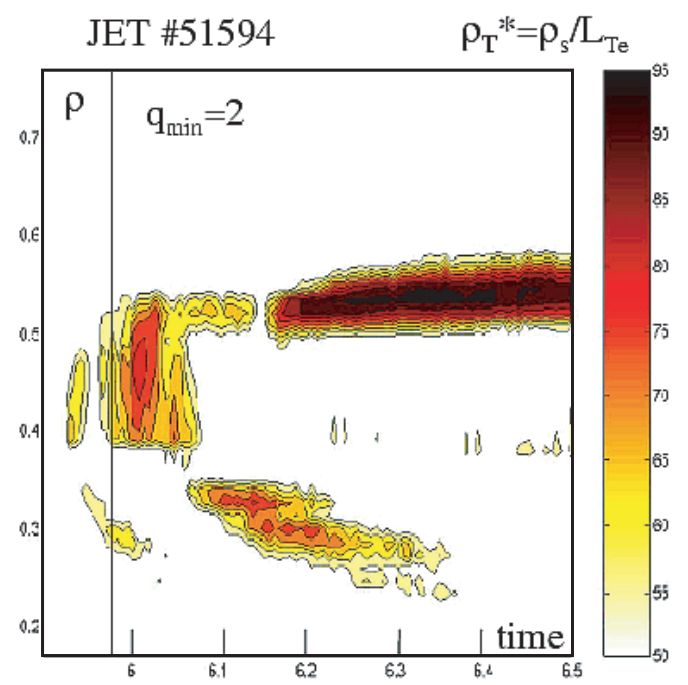

of density fluctuations is not always a good signature of ITB formation.

\section{Low order rational $q_{\min }$ and double internal barriers}

The favourable role of a low order rational value of the minimum safety factor has been long emphasized for in JET optimized shear plasmas [22,34]. This role has been confirmed recently in reversed shear plasmas thanks to the observation of Alfvèn wave cascades $[34,35]$. The $q$ profile in JET during a current ramp-up is such that $q_{\text {min }}$ decreases with time, crossing successively several low order rational surfaces. The case of $q_{\min }=2$ is intriguing and analysed in detail in a companion paper [35]. An example is shown in figure 6 that shows contour lines of $\rho_{\mathrm{T}}^{*}$ for pulse \#51573 [34]. First, a barrier appears at $R \approx 3.35 \mathrm{~m}$ in a region where the shear is negative. A dramatic change of structure appears at $t \approx 6 \mathrm{~s}$. This corresponds to the appearance of the surface $q=2$ at $q_{\min }$. Then two barriers appear that follow approximately the two $q=2$ surfaces. Clearly, most transport models can hardly predict this behaviour since they do not usually assign a special role to resonant surfaces. So this question deserves some attention.

A first explanation relies on MHD modes located at $q=2$ generating a localized velocity shear. A correlation between ITB formation and MHD activity was found in positive (optimized) shear plasmas [34]. On the other hand, no strong MHD activity is observed in reversed shear plasmas apart from the Alfvèn cascade itself. However, tearing modes located at $q=2$ surfaces may be difficult to detect. Turbulence itself could be responsible for a flow generation close to rational $q$ values. This explanation does receive some support from electromagnetic turbulence simulations with the CUTIE code [36]. These simulations also show that the bootstrap current is enhanced near rational $q$ values, thus further lowering the magnetic shear locally.

A second explanation relies on the existence of gaps in the density of low wave number rational surfaces. This gap is wider when $q_{\min }$ is close to a low order rational number.

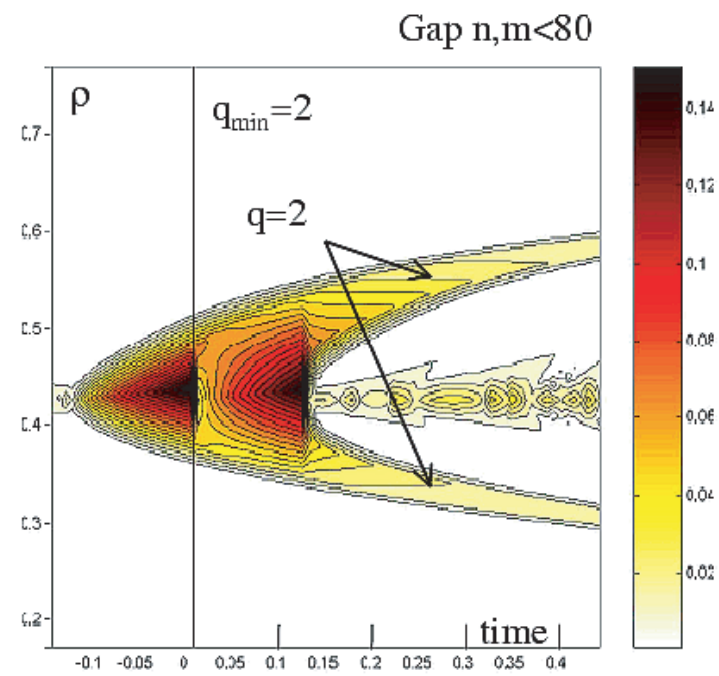

Figure 7. Contour lines of $\rho_{\mathrm{T}}^{*}$ (left panel) and of the distance (in metres) between adjacent resonant surfaces such that $n, m<80$ (right panel) (from [35]). 


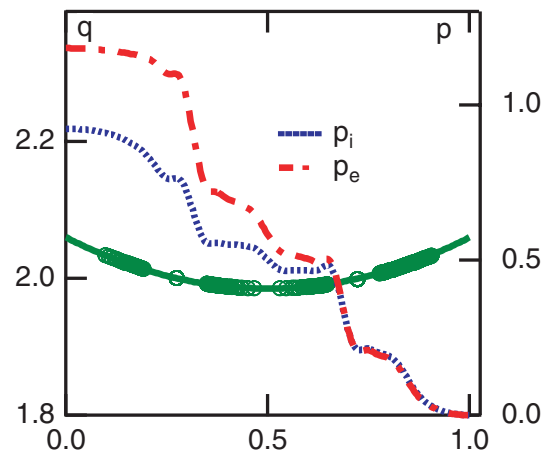

Figure 8. Simulation of a barrier with reversed magnetic shear and $q_{\text {min }}$ just below 2 with the turbulence code TRB. Circles are the position of resonant surfaces on the $q$ profile. The dashed lines are the ion and electron pressure profiles.

It depends sensitively on the curvature of the $q$ profile as illustrated by equation (1) [31,32]. Also, gaps tend to develop in the vicinity of low order rational numbers even for finite magnetic shear. Indeed, a correlation is found between the time-dependent position of the gaps and the actual evolution of a double barrier (figure 7, [33]). First, a large gap appears just before $q_{\min }=2$ (typically for $q_{\min }-2$ of the order of a few $10^{-3}$ ). Second, once $q_{\min }$ becomes smaller than 2 , two gaps follow the $q=2$ surfaces, whereas the central gap close to $q_{\min }$ contains high wave number resonant surfaces. It may, therefore, be possible that a strong barrier only appears when $q_{\min }$ crosses the $q=2$ surface, then splits. Coexistence of barriers is possible, as shown in figure 8 . The same figure shows that the barriers are stronger near $q=2$ than near $q_{\mathrm{min}}$. The notion of gaps in the density of resonant surfaces is close to the 'resonance-free' zones introduced by Brakel et al [37] to explain the dependence of the confinement in W7-AS on rational values of the safety factor. Note, however, that an explanation based on the density of rational surfaces does not explain the onset and self-sustainment of a barrier located somewhat in the negative shear region (as in \#51573 before $t=6 \mathrm{~s}$ ). The reversal of the precession frequency of trapped electrons for $s=-\frac{3}{8}$ (see equation (A4)) may explain the onset of this type of barrier. Thus, both $s<0$ and $s=0$ (and rational $q_{\min }$ ) must be invoked to explain the whole history of this kind of plasma.

\section{Conclusion}

Many experimental results in JET indicate that the onset of an ITB is sensitive to the profile of the safety factor. Part of these observations can be explained by the dependence of the linear growth rate on the safety factor and its gradient. Both linear stability analysis and turbulence simulations confirm this result. Models based on a transport reduction due to magnetic shear combined with velocity shear also reproduce the data in a satisfactory way. Whether it is zero or negative magnetic shear that matters remains to be clarified. Many barriers in JET appear at zero magnetic shear and low order rational $q_{\min }$. However, there also exist examples of electron transport barriers in the region of negative magnetic shear. Most transport models fail to explain the particular role of rational surfaces. Two explanations are possible for this special role. One is based on MHD or low $m, n$ turbulence modes generating a localized $E \times B$ shear flow. The second explanation relies on the development of a region without any low wave number resonant surface. Turbulence simulations confirm the possible coexistence of several barriers. They also indicate that rational $q$ surfaces play a special role.

\section{Appendix. Model implemented in the TRB code}

The following set of five fluid equations is used here to describe a collisionless ITG and TEM turbulence

$$
\begin{aligned}
d_{\mathrm{t}} n_{\mathrm{e}}= & \mathrm{i} \omega_{\mathrm{dte}}\left(n_{\mathrm{e}, \mathrm{eq}} \phi-p_{\mathrm{e}}\right)+S_{\mathrm{n}} \\
d_{\mathrm{t}} p_{\mathrm{e}}= & \mathrm{i} \omega_{\mathrm{dte}} \Gamma\left(n_{\mathrm{e}, \mathrm{eq}} \phi+T_{\mathrm{e}, \mathrm{eq}}^{2} n_{\mathrm{e}}-2 T_{\mathrm{e}, \mathrm{eq}} p_{\mathrm{e}}\right)+S_{\mathrm{pe}} \\
d_{\mathrm{t}} \Omega= & -n_{\mathrm{e}, \mathrm{eq}} \nabla_{\|} v_{\| \mathrm{i}}-\mathrm{i} \omega_{\mathrm{di}}\left(n_{\mathrm{e}, \mathrm{eq}} \phi+p_{\mathrm{i}}\right) \\
& -\mathrm{i} \omega_{\mathrm{dte}} f_{\mathrm{t}}\left(n_{\mathrm{e}, \mathrm{eq}} \phi-p_{\mathrm{e}}\right)+\left[p_{\mathrm{i}, \mathrm{eq}}, \nabla_{\perp}^{2} \phi\right] \\
& +f_{\mathrm{c}}\left[n_{\mathrm{e}, \mathrm{eq}}, \phi\right]
\end{aligned}
$$

where $n_{\mathrm{s}}, T_{\mathrm{s}}, p_{\mathrm{s}}, v_{\| \mathrm{s}}, \phi$ are the normalized density, temperature, pressure, parallel velocity and electric potential, respectively (the labels ' $e$ ' and ' $i$ ' are for electrons and ions, no impurity is included). The generalized vorticity $\Omega$ is defined as

$$
\Omega=n_{\mathrm{e}, \mathrm{eq}}\left[\frac{f_{\mathrm{c}}\left(\phi-\phi_{\mathrm{eq}}\right)}{T_{\mathrm{e}, \mathrm{eq}}}-\nabla_{\perp}^{2} \phi\right]
$$

The normalization is of the gyroBohm type,

$$
\begin{aligned}
& n_{\mathrm{e}} \rightarrow \frac{a}{\rho_{\mathrm{s} 0}} \quad \frac{n_{\mathrm{e}}}{n_{0}}, \quad p_{\mathrm{e}, \mathrm{i}} \rightarrow \frac{a}{\rho_{\mathrm{s} 0}} \quad \frac{p_{\mathrm{e}, \mathrm{i}}}{p_{0}}, \\
& \phi \rightarrow \frac{a}{\rho_{\mathrm{s} 0}} \quad \frac{e \phi}{T_{0}}, \quad v_{\| \mathrm{i}} \rightarrow \frac{a}{\rho_{\mathrm{s} 0}} \quad \frac{v_{\| \mathrm{i}}}{c_{\mathrm{s} 0}}
\end{aligned}
$$

where $\rho_{\mathrm{s} 0}$ is the ion gyroradius $\left(\rho_{\mathrm{s} 0}=m_{\mathrm{i}} c_{\mathrm{s} 0} / e_{\mathrm{i}} B_{0}, c_{\mathrm{s} 0}\right.$ is the sound speed $\left.\left(T_{0} / m_{\mathrm{i}}\right)^{1 / 2}\right), a$ and $R$ are the minor and major radii, respectively, $n_{0}, T_{0}, p_{0}=n_{0} T_{0}$ are reference values. Time and spatial coordinates are normalized to $a / c_{\mathrm{s} 0}$ and $\rho_{\mathrm{s} 0}$. The geometry of flux surfaces is circular concentric, $(r, \theta, \varphi)$ being the labels of the minor radius, poloidal and toroidal angles, respectively ( $\rho=r / a$ is the normalized minor radius). The fraction of trapped (resp. passing) electrons is $f_{\mathrm{t}}=2 / \pi(2 r / R)^{1 / 2}$ (resp. $\left.f_{\mathrm{c}}=1-f_{\mathrm{t}}\right)$. The electron precession drift and the ion curvature drift operators are, respectively,

$$
\omega_{\mathrm{dte}}=-\mathrm{i} 2 \varepsilon_{\mathrm{a}} \lambda_{\mathrm{t}} \rho_{\mathrm{s} 0} q r^{-1} \partial_{\varphi}, \quad \lambda_{\mathrm{t}}=\frac{1}{4}+\frac{2 s}{3}
$$

and

$$
\omega_{\mathrm{di}}=-\mathrm{i} 2 \varepsilon_{\mathrm{a}} \rho_{\mathrm{s} 0}\left(\cos (\theta) r^{-1} \partial_{\theta}+\sin (\theta) \partial_{\mathrm{r}}\right)
$$

The function $\lambda_{\mathrm{t}}=\frac{1}{4}+2 \mathrm{~s} / 3$ characterizes the dependence of the precession frequency on the magnetic shear $s=$ 
$\rho \mathrm{d} q / q \mathrm{~d} \rho$ and $\varepsilon_{\mathrm{a}}=a / R$ parametrizes the curvature. The Lagrangian time derivative is defined as $d_{\mathrm{t}}=\partial_{\mathrm{t}}+[\phi]-$,$D ,$ where $D$ is a 'collisional' diffusion operator and $[f, g]=$ $r^{-1}\left(\partial_{r} f \partial_{\theta} g-\partial_{\theta} f \partial_{r} g\right)$. The functions $S_{\mathrm{n}}, S_{\mathrm{v}}, S_{\mathrm{pe}}, S_{\mathrm{pi}}$ are particle, momentum, ion and electron heat sources, respectively. The label 'eq' indicates a flux average. Note that the perturbed part of $f_{\mathrm{t}} n_{\mathrm{e}}$ is the fluctuating density of trapped electrons, whereas $\hat{n}_{\mathrm{e}}$ is the total equilibrium electron density. The adiabatic compression index is $\Gamma=\frac{5}{3}$. The vorticity equation (A1c) expresses an ambipolarity condition. The vorticity is coupled via the curvature drifts to electron and ion pressure, which are governed by equations (A1b) and $(\mathrm{A} 1 e)$. This coupling is responsible for TEM and toroidal ITG instabilities, while the coupling with the parallel momentum equation (A1d) is responsible for the slab ITG instability. A highly simplified (but useful) estimate of the growth rate can be obtained by assuming low wave numbers $\left(k_{\perp} \rho_{\mathrm{i}} \ll 1\right)$, strongly ballooned modes $(\theta \ll 1)$, large pressure gradient and no density gradient, and pure convection of the electron and ion pressure $d_{\mathrm{t}} p=0$, namely

$$
\gamma_{0}^{2}=f_{\mathrm{t}} \omega_{\mathrm{dte}} \omega_{\mathrm{pe}}^{*}+\omega_{\mathrm{di}} \omega_{\mathrm{pi}}^{*}
$$

where

$\omega_{\mathrm{pe}}^{*}=\frac{-n q}{r} \rho_{s 0} \frac{a \partial_{r} p_{\mathrm{e}, \mathrm{eq}}}{n_{\mathrm{e}, \mathrm{eq}}}, \quad \omega_{\mathrm{pi}}^{*}=\frac{n q}{r} \rho_{s 0} \frac{a \partial_{r} p_{\mathrm{i}, \mathrm{eq}}}{n_{\mathrm{e}, \mathrm{eq}}}$

and $n$ is the toroidal wave number. A subset of equations describing ITG modes is obtained by setting $f_{\mathrm{t}}=0$ (no trapped electrons)

\section{References}

[1] Drake J.F. et al 1996 Phys. Rev. Lett. 77494

[2] Romanelli F. and Zonca F. 1993 Phys. Fluids B 54081

[3] Waltz R.E., Kerbel G.D., Milovitch J. and Hammett G.W. 1995 Phys. Plasmas 22408

[4] Biglari H., Diamond P. and Terry P.W. 1990 Phys. Fluids B 21

[5] Hahm T.S. and Burrell K.H. 1995 Phys. Plasmas 21648

[6] Hamaguchi S. and Horton W. 1992 Phys. Fluids B 4319

[7] Weiland J. 2000 Collective Modes in Inhomogeneous Plasmas (Bristol: Institute Of Physics Publishing)
[8] Tresset G. et al 2002 Nucl. Fusion 42520

[9] Mantica P. et al 2002 Transient heat transport studies in jet conventional and advanced tokamak plasmas 19th International Conf. on Plasma Physics and Controlled Nuclear Fusion Research (Lyon, 2002) (Vienna: IAEA) IAEA-CN-94/EX/P1-04

[10] Wolf R.C. et al Characterisation of ion heat conduction in JET and ASDEX-Upgrade plasmas with and without internal transport barriers Plasma Phys. Control. Fusion submittted

[11] Baranov Y. Private communication (Culham)

[12] Budny R.V. et al 2002 Plasma Phys. Control. Fusion 441215

[13] Crisanti F. et al 2001 Nucl. Fusion 41883

[14] Eriksson L.-G. et al 2002 Phys. Rev. Lett. 88145001

[15] Rogister A.L. 2001 Nucl. Fusion 411101

[16] Kotschenreuther M. et al 1995 Phys. Plasmas 22381

[17] Newman D.E. et al 1998 Phys. Plasmas 5938

[18] Bourdelle C. et al 2002 Nucl. Fusion 42892

[19] Houlberg W.A., Shaing K.C., Hirshman S.P. and Zarnstorff M.C. Phys. Plasmas 43230

[20] Kim J. et al 1994 Phys. Rev. Lett. 722199

[21] Maget P., Esposito B. and Joffrin E. et al 2003 Plasma Phys. Control. Fusion 451385

[22] Challis C. et al 2002 Plasma Phys. Control. Fusion 441031

[23] Hogeweij G.M.D. et al 2002 Plasma Phys. Control. Fusion 44 1155

[24] Conway G.D. et al 2002 Plasma Phys. Control. Fusion 44 1167

[25] Esposito B. et al 2003 Plasma Phys. Control. Fusion 45933

[26] Tala T.J.J. et al 2001 Plasma Phys. Control. Fusion 43507

[27] Imbeaux F. et al 2002 Analytical solutions for the propagation of heat pulses with temperature gradient length dependent diffusion coefficient EU-US TTF Meeting (Cordoba, 2002)

[28] Zhu P., Bateman G., Kritz A.H. and Horton W. 2000 Phys. Plasmas 72898

[29] Tala T.J.J. et al 2002 Plasma Phys. Control. Fusion 44 A495

[30] Litaudon X., Crisanti F. and Alper B. 2002 Plasma Phys. Control. Fusion 441057

[31] Garbet X. et al 2001 Phys. Plasmas 82793

[32] Voitsekhovitch I. et al 2002 Phys. Plasmas 94671

[33] Beyer P., Benkadda S., Garbet X. and Diamond P.H. 2000 Phys. Rev. Lett. 854892

[34] Joffrin E. et al 2002 Plasma Phys. Control. Fusion 441739

[35] Joffrin E. et al Internal transport barrier triggering by rational magnetic flux surfaces in tokamaks Nucl. Fusion. submitted

[36] Thyagaraja A. 2000 Plasma Phys. Control. Fusion 42 B255

[37] Brakel R. et al 1997 Plasma Phys. Control. Fusion 39 B273 